\title{
Compressive sensing-based angle estimation for MIMO radar with multiple snapshots
}

\author{
Jianfeng $\mathrm{Li}^{1}$, Xiaofei Zhang ${ }^{1,2}$, Tong $\mathrm{Hu}^{1}$ \\ 1. Department of Electronic Engineering, Nanjing University of Aeronautics \& Astronautics, Nanjing, China \\ 2. Nanjing Panda Electronics Group, Nanjing, China \\ e-mail: lijianfengtin@126.com
}

\begin{abstract}
The issue of angle estimation for multiple-input multiple-output (MIMO) radar is studied and an algorithm for the estimation based on compressive sensing with multiple snapshots is proposed. The dimension of received signal is reduced to make the computation burden lower, and then the noise sensitivity is reduced by the eigenvalue decomposition (EVD) of the covariance matrix of the reduced-dimensional signal. Finally the signal subspace obtained from the eigenvectors is realigned to apply the orthogonal matching pursuit (OMP) for angle estimation. The angle estimation performance of the proposed algorithm is better than that of estimation of signal parameters via rotational invariance techniques (ESPRIT) algorithm, and reduceddimension Capon. Furthermore, the proposed algorithm works well for coherent targets, and requires no knowledge of the noise. The complexity analysis and simulation results verify the effectiveness of the algorithm.
\end{abstract}

Keywords- multiple-input multiple-output (MIMO) radar; angle estimation; compressive sensing; $\mathrm{OMP}$

\section{INTRODUCTION}

Multiple-input multiple-output (MIMO) radars use multiple antennas to simultaneously transmit diverse waveforms and utilize multiple antennas to receive the reflected signals, and they have many potential advantages over conventional phased-array radars [1-4]. MIMO radar systems have more degrees of freedom than other systems with a single transmit antenna, and these additional degrees of freedom can overcome fading effect, enhance spatial resolution, strengthen parameter identifiability and improve target detection performance [5-8]. Angle estimation is a key issue in MIMO radar, and several algorithms for angle estimation have been established, which contain estimation of signal parameters via rotational invariance techniques (ESPRIT) algorithm [9], Capon algorithm [10], multiple signal classification (MUSIC) algorithm [11] and parallel factor analysis algorithms [12-13]. Reduced-dimension (RD) ESPRIT algorithm [14] and RD Capon [15] have been proposed for angle estimation in monostatic MIMO radar, and they reduce the complexity and improve the performance compared to conventional methods.

Compressive sensing [16-17] has attracted a lot of attention in the last decade, and it has been applied to a variety of problems, including image reconstruction and restoration, wavelet denoising, feature selection in machine learning, radar imaging and penalized regression [18]. The super-resolution property and ability of resolving coherent sources can be achieved when apply it in the source location
[19]. Lots of the location methods using compressive sensing just use one snapshot and are very sensitive to the noise, for multiple snapshots, $\ell_{1}$-SVD method [18] employs $\ell_{1}$ norm to enforce sparsity and singular value decomposition to reduce complexity and sensitivity to noise, and sparse recovery for weighted subspace fitting (SRWSF) [19] improved the $\ell_{1}$-SVD method via the weight to the subspace. However, both the method in [18] and [19] have a common problem, which is the choice of the regularization parameter, so a prior knowledge of the noise may be known.

In this paper, we propose a compressive sensing-based method for angle estimation in MIMO radar. The reduceddimension transformation is utilized to reduce the dimension of the signal, i.e. the dimension of the dictionary, and then the eigenvalue decomposition (EVD) is employed to reduce the sensitivity of the noise. Finally, according to the relationship between the direction matrix and signal subspace, the signal subspace is realigned to apply the orthogonal matching pursuit (OMP) [20] for angle estimation. The angle estimation performance of the proposed algorithm is better than that of RD ESPRIT algorithm, and RD Capon. Furthermore, the proposed algorithm works well for coherent targets, and requires no knowledge of the noise.

Notation: $(.)^{T} 、(.)^{H} 、(.)^{-1}$ and $(.)^{+}$denote transpose, conjugate-transpose, inverse, pseudo-inverse operations, respectively; $\operatorname{diag}(\mathbf{v})$ stands for diagonal matrix whose diagonal element is a vector $\mathbf{v} ; D_{n}($.$) is to take the nth row$ of the matrix to construct a diagonal matrix; $\mathbf{I}_{K}$ is a $K \times K$ identity matrix; $\otimes$, $\circ$ and $\odot$ are the Kronecker product, Khatri-Rao product and Hadamard product, respectively; $\operatorname{Re}($.$) is to get real part of the complex; \min ($.$) is to get$ minimum elements of an array; $E[$.] is expectation operator and $\operatorname{vec}(\cdot)$ denotes an operator stacking the columns of a matrix on top of each other

\section{DATA MODEL}

We consider a monostatic MIMO radar system equipped with both of uniform linear arrays for the transmit and receive arrays, and the transmit array and receive array are both located in the y-axis with half-wavelength spacing between adjacent antennas, respectively. We assume that there are $\mathrm{K}$ targets in the $\mathrm{y}-\mathrm{z}$ plane, and the output of the matched filters at the receiver can be expressed as

$$
\mathbf{x}(t)=\left[\mathbf{a}_{r}\left(\theta_{1}\right) \otimes \mathbf{a}_{t}\left(\theta_{1}\right), \cdots, \mathbf{a}_{r}\left(\theta_{K}\right) \otimes \mathbf{a}_{t}\left(\theta_{K}\right)\right] \mathbf{s}(t)+\mathbf{n}(t)
$$


where $\theta_{k}$ is the (Direction of arrival) DOA of the kth target with respect to the transmit array normal or the receive array normal; $\mathbf{s}(t)=\left[s_{1}(t), s_{2}(t), \cdots, s_{K}(t)\right]^{T} \in \mathbb{C}^{K \times 1}, s_{k}(t)=\beta_{k} e^{j 2 \pi f_{k} t}$ with $f_{k}$ being Doppler frequency and $\beta_{k}$ the RCS; $\mathbf{n}(t)$ is an $M N \times 1$ Gaussian white noise vector of zero mean and covariance matrix $\sigma^{2} \mathbf{I}_{M N} ; \mathbf{a}_{r}\left(\theta_{k}\right) \otimes \mathbf{a}_{t}\left(\theta_{k}\right)$ is the Kronecker product of the receive and the transmit steering vectors for the kth target, and

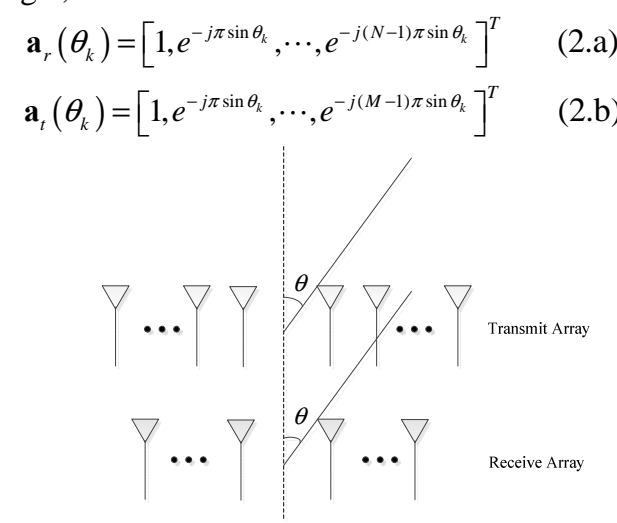

Figure 1. Array structure of monostatic MIMO radar

III. COMPRESSIVE SENSING-BASED METHOD FOR ANGLE ESTIMATION IN MIMO RADAR WITH MULTIPLE SNAPSHOTS

\section{A. Reduced-dimension transformation}

The length of $\mathbf{a}_{r}\left(\theta_{k}\right) \otimes \mathbf{a}_{t}\left(\theta_{k}\right)$ is $\mathrm{MN}$, which costs high computation in the later recovery via OMP, so the reduceddimension transformation is necessary. As

$$
\mathbf{a}\left(\theta_{k}\right)=\mathbf{a}_{r}\left(\theta_{k}\right) \otimes \mathbf{a}_{t}\left(\theta_{k}\right)=\mathbf{G b}\left(\theta_{k}\right)
$$

where

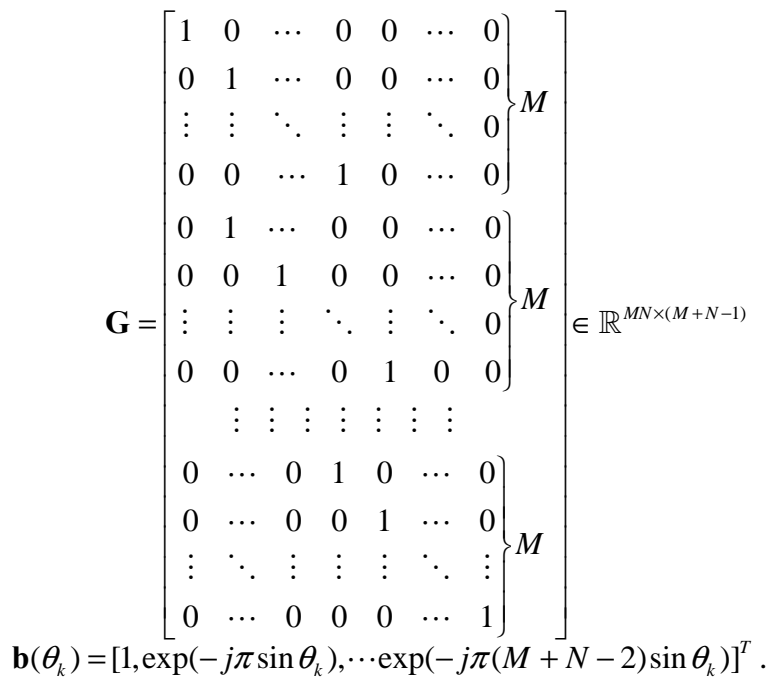

Then we define $\mathbf{W} \triangleq \mathbf{G}^{H} \mathbf{G}$,

$$
\mathbf{W}=\operatorname{diag}(1,2, \cdots, \underbrace{\min (M, N), \cdots, \min (M, N)}_{|M-N|+1}, \cdots, 2,1)
$$

Using the reduced-dimension transformation $\mathbf{W}^{-\frac{1}{2}} \mathbf{G}^{H}$ for the receive signal $\mathbf{x}(t)$, we obtain

$$
\begin{aligned}
\mathbf{y}(t) & =\mathbf{W}^{-\frac{1}{2}} \mathbf{G}^{H} \mathbf{x}(t) \\
& =\mathbf{W}^{-\frac{1}{2}} \mathbf{W}\left[\mathbf{b}\left(\theta_{1}\right), \mathbf{b}\left(\theta_{2}\right), \cdots, \mathbf{b}\left(\theta_{K}\right)\right] \mathbf{s}(t)+\mathbf{W}^{-\frac{1}{2}} \mathbf{G}^{H} \mathbf{n}(t)(6) \\
& =\mathbf{W}^{\frac{1}{2}} \mathbf{B} \mathbf{s}(t)+\mathbf{W}^{-\frac{1}{2}} \mathbf{G}^{H} \mathbf{n}(t)
\end{aligned}
$$

where $\mathbf{B}=\left[\mathbf{b}\left(\theta_{1}\right), \mathbf{b}\left(\theta_{2}\right), \cdots, \mathbf{b}\left(\theta_{K}\right)\right] \in \mathbb{C}^{(M+N-1) \times K} . \mathbf{W}^{\frac{1}{2}} \mathbf{B}$ can be regarded as the new direction matrix which has lower dimension, and the reduced-dimension matrix is sparse, its transformation adds less computational load. The covariance matrix of $\mathbf{y}(t)$ in (6) is $\mathbf{R}_{y}(t) \in \mathbb{C}^{(M+N-1) \times(M+N-1)}$

$$
\begin{aligned}
& \mathbf{R}=E\left[\mathbf{y}(t) \mathbf{y}^{H}(t)\right] \\
& =\mathbf{W}^{\frac{1}{2}} \mathbf{B} \mathbf{R}_{s} \mathbf{B}^{H} \mathbf{W}^{\frac{1}{2}}+\sigma^{2} \mathbf{I}_{M+N-1}
\end{aligned}
$$

where $\mathbf{R}_{s}=E\left[\mathbf{s}(t) \mathbf{s}^{H}(t)\right]$.

\section{B. Compressive sensing-based method for angle estimation}

The covariance matrix in (7) can be decomposed as

$$
\mathbf{R}=\mathbf{E}_{s} \mathbf{D}_{s} \mathbf{E}_{s}^{H}+\mathbf{E}_{n} \mathbf{D}_{n} \mathbf{E}_{n}^{H}
$$

where $\mathbf{D}_{s}$ denotes a $K \times K$ diagonal matrix formed by $K$ largest eigen-values, and $\mathbf{D}_{n}$ denotes a diagonal matrix formed by the rest $(M+N-1)-K$ smaller eigen-values. $\mathbf{E}_{s}$ and $\mathbf{E}_{n}$ represent the signal subspace and noise subspace, respectively, of which $\mathbf{E}_{s}$ stands for the eigenvectors corresponding to the $\mathrm{K}$ largest eigen-values, $\mathbf{E}_{n}$ consists of the rest eigenvectors. The equation between $\mathbf{E}_{s}$ and the direction matrix can be formulated as

$$
\mathbf{E}_{s}=\mathbf{W}^{\frac{1}{2}} \mathbf{B T}
$$

where $\mathbf{T}$ represents a nonsingular $K \times K$ matrix.

Let $\tilde{\theta}_{1}, \tilde{\theta}_{2}, \cdots, \tilde{\theta}_{L}$ be a sampling grid of all target locations of interest. The number of potential source locations will typically be much greater than the number of sources $K$ or even the number of sensors $M+N-1$. We construct a matrix composed of steering vectors corresponding to each potential target location as its columns: $\boldsymbol{\Theta}=\left[\mathbf{b}\left(\tilde{\theta}_{1}\right), \mathbf{b}\left(\tilde{\theta}_{2}\right), \cdots, \mathbf{b}\left(\tilde{\theta}_{L}\right)\right]$.

Then construct a matrix $\mathbf{Q} \in \mathbb{C}^{L \times K}$, and the rows of $\mathbf{Q}$ corresponding to the true DOAs keep the same with those of $\mathbf{T}$, with the other rows being all-zero. And

$$
\mathbf{E}_{s}=\mathbf{W}^{\frac{1}{2}} \mathbf{\Theta Q}
$$

This implies that if $\mathbf{Q}$ can be recovered from $\mathbf{E}_{s}$, the DOAs can be determined by exploiting the positions of nonzero rows of $\mathbf{Q}$. Define $\mathbf{e}_{s}=\operatorname{vec}\left(\mathbf{E}_{s}\right)$, which is the realigned form of $\mathbf{E}_{s}$, and it satisfies

$$
\mathbf{e}_{s}=\left(\mathbf{I}_{K} \otimes\left(\mathbf{W}^{\frac{1}{2}} \boldsymbol{\Theta}\right)\right) \operatorname{vec}(\mathbf{Q})=\mathbf{\Phi q}
$$


where $\quad \boldsymbol{\Phi}=\mathbf{I}_{K} \otimes\left(\mathbf{W}^{\frac{1}{2}} \boldsymbol{\Theta}\right) \in \mathbb{C}^{K(M+N-1) \times L K} \quad, \quad$ and $\mathbf{q}=\operatorname{vec}(\mathbf{Q}) \in \mathbb{C}^{L K \times 1}$ is a sparse vector with $K^{2}$ nonzero elements.

According to (11), $\boldsymbol{\Phi}$ can be regard as the recovery matrix or dictionary in compressive sensing, and the sparse vector $\mathbf{q}$ can be obtained by utilizing OMP recovery method [20]. The detailed recovery processing via OMP is shown in Fig.2. Then $\mathbf{Q}$ can be estimated by reshaping the vector $\mathbf{q}$ into the $L \times K$ matrix and the nonzero rows in $\mathbf{Q}$ will show the DOAs of the targets.

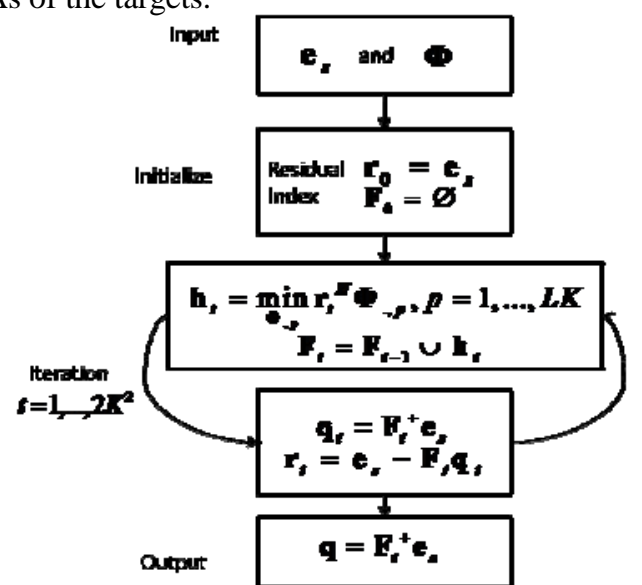

Figure 2. The OMP algorithm flow

With respect to (7), the covariance matrix $\mathbf{R}=E\left[\mathbf{y}(t) \mathbf{y}^{H}(t)\right]$ can be estimated with J snapshots by

$$
\hat{\mathbf{R}}=\frac{1}{J} \sum_{t=1}^{J} \mathbf{y}(t) \mathbf{y}^{H}(t)
$$

\section{Complexity analysis and CRB}

The proposed algorithm has higher complexity than RD ESPRIT, but has much lower complexity than RD Capon algorithm, which needs peak searching.

Fig. 3 shows the run time of the three algorithms in computer versus the number of antennas, we choose $\mathrm{M}=\mathrm{N}$ for simplify. From Fig.3, we find that our algorithm has much lower complexity than the RD Capon, and the change trend versus the number of antennas of the proposed algorithm is smaller than that of the other two algorithms.

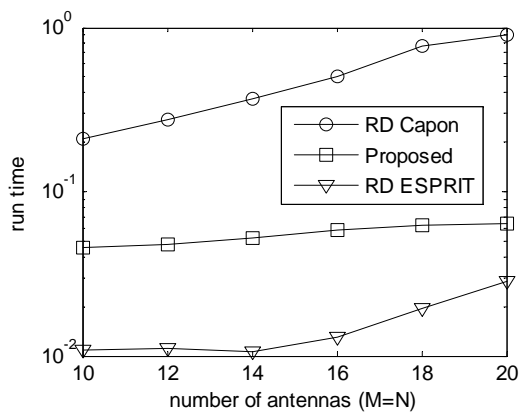

Figure 3. complexity comparison against number of antennas
According to Ref.[21], we can derive the CRB in bistatic MIMO radar

$$
C R B=\frac{\sigma^{2}}{2 J}\left\{\operatorname{Re}\left[\mathbf{D}^{H} \boldsymbol{\Pi}_{\mathbf{A}}^{\perp} \mathbf{D} \odot \hat{\mathbf{P}}_{w}{ }^{T}\right]\right\}^{-1}
$$

where

$$
\mathbf{D}=\left[\frac{\partial \mathbf{a}_{1}}{\partial \theta_{1}}, \frac{\partial \mathbf{a}_{2}}{\partial \theta_{2}}, \ldots, \frac{\partial \mathbf{a}_{K}}{\partial \theta_{K}}, \frac{\partial \mathbf{a}_{1}}{\partial \phi_{1}}, \frac{\partial \mathbf{a}_{2}}{\partial \phi_{2}}, \ldots, \frac{\partial \mathbf{a}_{K}}{\partial \phi_{K}}\right] \quad \text { with }
$$

$\mathbf{a}_{k}=\mathbf{a}_{r}\left(\theta_{k}\right) \otimes \mathbf{a}_{t}\left(\theta_{k}\right)$, and $\mathbf{A}=\left[\mathbf{a}_{1}, \cdots, \mathbf{a}_{K}\right] ; \quad \hat{\mathbf{P}}_{w}=\left[\begin{array}{ll}\hat{\mathbf{P}}_{s} & \hat{\mathbf{P}}_{s} \\ \hat{\mathbf{P}}_{s} & \hat{\mathbf{P}}_{s}\end{array}\right]$,

$\hat{\mathbf{P}}_{s}=\frac{1}{J} \sum_{t=1}^{J} \mathbf{s}(t) \mathbf{s}^{H}(t) ; \boldsymbol{\Pi}_{\mathbf{A}}^{\perp}=\mathbf{I}_{M N}-\mathbf{A}\left(\mathbf{A}^{H} \mathbf{A}\right)^{-1} \mathbf{A}^{H}$.

\section{SiMULATION RESULTS}

Define root mean square error (RMSE) as $\frac{1}{K} \sum_{k=1}^{K}\left(\frac{1}{1000} \sum_{l=1}^{1000}\left[\left(\hat{\theta}_{k, l}-\theta_{k}\right)^{2}\right]\right)^{1 / 2}$, where $\hat{\theta}_{k, l}$ is the estimate of DOD $\theta_{k}$ of the lth Monte Carlo trial. We assume there are $K$ $=2$ targets with angle $\left(\theta_{1}, \theta_{2}\right)=\left(5^{\circ}, 25^{\circ}\right)$. The RCSs and Doppler frequencies are $\left(\beta_{1}, \beta_{2}\right)=\left[e^{j \pi / 5}, 0.8 e^{j \pi / 4}\right]$ and $\left(f_{1}, f_{2}\right)=[100 \mathrm{~Hz}, 500 \mathrm{~Hz}]$, respectively.

Fig.4 depicts angle estimation result of the proposed algorithm for all two targets with $M=16, N=14, J=50$, SNR(Signal-to-Noise Ratio) $=0 \mathrm{~dB}$, respectively. It is shown that the DOAs can be clearly observed.

We compare the proposed algorithm against the $\mathrm{RD}$ ESPRIT algorithm, RD Capon algorithm and CRB. Fig. 5 presents the comparison of the algorithms. From Fig. 5, we can find that the angle estimation performance of the proposed algorithm is better than that of RD ESPRIT algorithm, and has better performance than RD Capon algorithm when SNR is higher.

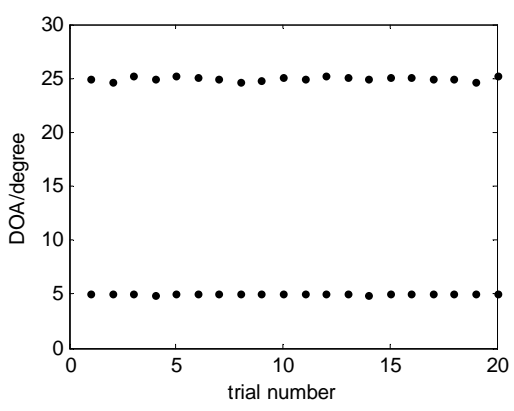

Figure 4. Angle estimation result of the proposed algorithm

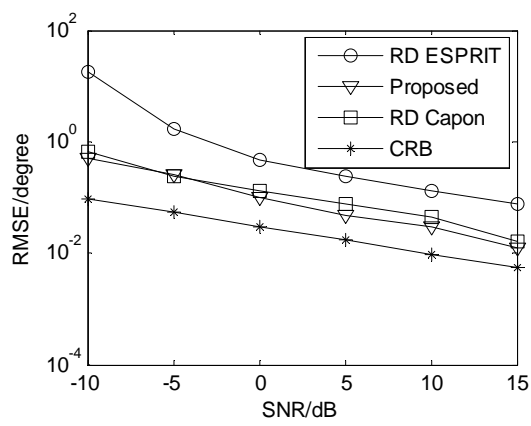

Figure 5. Angle estimation performance comparison 


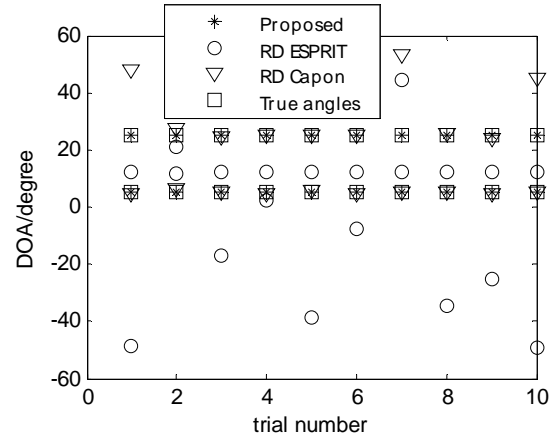

Figure 6. Angle estimation results for coherent targets

Fig.6 shows the estimation results of the three algorithms with two coherent targets $\left(\left(\beta_{1}, \beta_{2}\right)=\left[e^{j \pi / 5}, 0.8 e^{j \pi / 5}\right]\right.$ and $f_{1}=f_{2}=500 \mathrm{~Hz}$ ), and $\mathrm{SNR}=10 \mathrm{~dB}$. It can be indicated from Fig. 6 that the proposed algorithm works well for coherent targets, which make the other two algorithms fail to work or have performance degradation.

\section{CONCLUSION}

In this paper, we have proposed a DOA estimation algorithm in monostatic MIMO radar using compressive sensing and multiple snapshots. By using the reduceddimensional transformation, EVD of the data and OMP for recovery, the angle estimation performance of the proposed algorithm is better than that of RD ESPRIT and RD Capon and the algorithm is effective due to the lower complexity. Furthermore, the proposed algorithm works well for coherent targets, and requires no knowledge of the noise.

\section{ACKNOWLEDGMENT}

This work is supported by China NSF Grants (61271327,61071164), Jiangsu Planned Projects for Postdoctoral Research Funds (1201039C), China Postdoctoral Science Foundation (2012M521099), Open project of key laboratory of underwater acoustic communication and marine information technology (Xiamen University), Hubei Key Laboratory of Intelligent Wire1ess Communications (IWC2012002), Open project of Key Laboratory of Nondestructive Testing (Nanchang Hangkong University), Open project of Key Laboratory of modern acoustic of Ministry of Education (Nanjing University), the Aeronautical Science Foundation of China (20120152001), by Qing Lan Project and the Fundamental Research Funds for the Central Universities (NZ2012010, kfjj120115, kfjj20110215).

\section{REFERENCES}

[1] E. Fishler, A. Haimovich, R. S. Blum, L. J. Cimini, D. Chizhik, and R. A. Valenzuela, "MIMO radar: an idea whose time has come", in Proc. IEEE Radar Conf., Apr. 2004: 71-78.
[2] J. Li and P. Stoica, "MIMO radar-diversity means superiority”, in Proc.14th Adaptive Sensor Array Process. Workshop (ASAP '06), Lincoln Lab, Mass, USA, Dec. 2006:1-6.

[3] X. Li, Z. Zhang et al, "A Study of Frequency Diversity MIMO Radar Beamforming”, IEEE 10th International Conference (ICSP2010), 2010:352-356

[4] R. Sharma, "Analysis of MIMO radar ambiguity functions and implications on clear region”, IEEE Radar Conf, June 2010:544-548.

[5] J. Li, G. Liao, H. Griffiths, "Bistatic MIMO Radar Space-Time Adaptive Processing”, IEEE International Radar Conference,Westin Crown Center in Kansas City, Missouri, May 2011:498-502.

[6] X.H. Wu, A.A. Kishk, A.W. Glisson, "MIMO-OFDM radar for direction estimation” IET Radar, Sonar \&Navigation, 2010, 4(1):2836.

[7] J. Li, P. Stoica, L. Xu, and W. Roberts, “On parameter identifiability of MIMO radar”, IEEE Signal Process. Lett, 2007, 14(12):968-971.

[8] J. Li, G. Liao, K. Ma and C. Zeng, "Waveform Decorrelation for Multitarget Localization in Bistatic MIMO Radar Systems”, 2010 IEEE International Radar Conference, Washington, May 2010:21-24.

[9] J. Chen, H. Gu and W. Su, "Angle estimation using ESPRIT without pairing in MIMO radar”, Electron. Lett., 2008, 44(24):1422-1423.

[10] H. Yan, J. Li, G. Liao, "Multitarget identification and localization using bistatic MIMO radar systems", EURASIP J. Adv. Signal Process., Article ID283483, 2008:1-8.

[11] X. Gao, X. Zhang, G. Feng, et al, "On the MUSIC-derived Approaches of Angle Estimation for Bistatic MIMO Radar”, WNIS '09. International Conference on Wireless Networks and Information Systems, 2009:343-346.

[12] X. Zhang, Z. Xu, L. Xu, D. Xu, "Trilinear decomposition-based transmit angle and receive angle estimation for multiple-input multiple-output radar”, IET Radar, Sonar \& Navigation, 2011,5(6): $626-631$.

[13] D. Nion, N.D. Sidiropoulos, “Adaptive algorithms to track the PARAFAC decomposition of a third-order tensor”, IEEE Trans. on Signal Proces., Jun. 2009, 57(6):2299-2310.

[14] X. Zhang and D. Xu, "Low-complexity ESPRIT-based DOA estimation for collocated MIMO radar using reduced-dimension transformation”, Electronics Letters, 2011, 47(4): 283-284.

[15] X. Zhang, Y. Huang, C. Chen, et.al. "Reduced-complexity Capon for direction of arrival estimation in a monostatic multiple-input multiple-output radar”, IET Radar, Sonar \& Navigation, 2012,6(8): 796-801.

[16] D. L. Donoho. Compressed sensing[J].IEEE Transactions on Information Theory, 2006, 52(4) : 1289-1306.

[17] E. Candes, J. Romberg, J. Tao. Robust uncertainty principles : Exact signal reconstruction from highly incomplete frequency information[J].IEEE Transactions on In formation Theory, 2006, 52(2) : 489-509.

[18] D. Malioutov, M. Cetin, and A. S. Willsky. "A sparse signal reconstruction perspective for source localization with sensor arrays". IEEE Trans. Signal Process. , 2005, 53, (8):. 3010 - 3022

[19] N. Hu, Z. Ye, D. Xu and S. Cao. "A sparse recovery algorithm for DOA estimation using weighted subspace fitting”, Signal processing, 2012, 92: 2566-2570.

[20] J. A. Tropp, and A. C. Gilbert. "Signal Recovery From Random Measurements Via Orthogonal Matching Pursuit”, IEEE Trans. Inf. Theory , 2007, 53, (12): 4655 - 4666.

[21] P. Stoica and A. Nehorai, "Performance study of conditional and unconditional direction-of-arrival estimation”, IEEE Trans. Signal Process., 1990, 38(10): 1783-1795. 